\title{
Imbalanced antiferromagnet in an optical lattice
}

\author{
Arnaud Koetsier, ${ }^{*}$ F. van Liere, and H. T. C. Stoof \\ Institute for Theoretical Physics, Utrecht University, Leuvenlaan 4, 3584 CE Utrecht, The Netherlands
}

(Received 3 August 2009; published 26 February 2010)

\begin{abstract}
We study the rich properties of the imbalanced antiferromagnet in an optical lattice. We present its phase diagram, discuss spin waves, and explore the emergence of topological excitations in two dimensions, known as merons, which are responsible for a Kosterlitz-Thouless transition that has never unambiguously been observed.
\end{abstract}

DOI: 10.1103/PhysRevA.81.023628

PACS number(s): 03.75.Lm, 75.30.Kz, 03.75.Ss, 05.50.+q

\section{INTRODUCTION}

Owing to their exquisite experimental tunability, ultracold atomic gases have become a test bed for many paradigmatic ideas in quantum many-body physics. In particular, ultracold atoms confined in an optical lattice (i.e., a periodic potential for neutral atoms created by orthogonal retroreflected laser beams) are accurately described by the Hubbard model. The narrow energy bands resulting from the periodic potential quench the kinetic energy of the atoms with respect to their interaction energy, enabling the exploration of strongly correlated phases that play a significant role in condensed-matter physics. The single-band Hubbard model is realized by cold atoms when the lattice potential is sufficiently strong that only the lowest-energy band is populated [1]. At a filling corresponding to one particle per lattice site, this model has a Mott-insulator phase for repulsive interactions and in an atomic Bose gas the theoretically predicted superfluid-to-Mott-insulator phase transition [2] was indeed observed experimentally [3]. For bosons, one commonly refers to this model as the BoseHubbard model. The Fermi-Hubbard model, referred to simply as the Hubbard model, was also realized experimentally [4] and the fermionic Mott-insulator has only recently been seen $[5,6]$.

Aside from being rich in novel physics in itself, fermions in an optical lattice may also shed new light on the poorly understood phenomenon of high-temperature superconductivity in the cuprates. There, the Hubbard model is thought to describe electrons in the periodic ion-lattice potential of the copper-oxygen planes, which are believed to undergo a quantum phase transition to a $d$-wave superconducting state as the filling fraction is reduced by doping. In this case, electrons on the same lattice site repel each other due to the Coulomb interaction. We therefore consider here the repulsive Hubbard model, although the attractive Hubbard model can also be realized and possesses interesting Bose-Einstein condensation (BEC)-BCS crossover physics [7].

While achieving the antiferromagnetic ground state of the Hubbard model in an atomic Fermi gas is currently a major experimental goal, another prominent topic that is the focus of a number of recent ground-breaking experiments is imbalance in two-component Fermi gases [8,9]. There the population of each spin species can be controlled permitting the exploration of uncharted imbalanced phases of great interest in condensed-matter, nuclear, high-energy, and astroparticle physics. In this article, we explore the bridge between these

\footnotetext{
*a.o.koetsier@uu.nl
}

two exciting directions in the physics of ultracold atomic gases by considering an imbalanced Fermi gas in an optical lattice at nonzero temperature. In agreement with previous work at zero temperature [10], we find that the imbalanced system exhibits a canted phase. We show that this produces both ferro and antiferromagnetic properties with gapped and gapless spin waves and demonstrate that topological excitations known as merons play an important role in the two-dimensional case, giving rise to a Kosterlitz-Thouless phase transition.

\section{PHASE DIAGRAM}

In the Mott-insulator phase only spin degrees of freedom remain. Low-lying excitations are then effectively described by the Heisenberg model with the Hamiltonian $\hat{H}=$ $(J / 2) \sum_{\langle i, j\rangle} \hat{\mathbf{S}}_{i} \cdot \hat{\mathbf{S}}_{j}$, where $\hbar \hat{\mathbf{S}}_{i}$ is the spin-1/2 operator on site $i,\langle i, j\rangle$ denotes a sum over nearest-neighbor sites of the bipartite hypercubic lattice of interest here. The exchange constant $J=4 t^{2} / U$ arises from the superexchange mechanism where $t$ is the tunneling amplitude and $U>0$ is the on-site Hubbard interaction energy in the lattice. That is, the system can lower its energy by virtual nearest-neighbor hops only when there is antiferromagnetic ordering. Note that the total spin operator $\sum_{i} \hat{\mathbf{S}}_{i}$ commutes with $\hat{H}$ and thus the magnetization is a constant of the motion.

The three-dimensional phase diagram calculated within mean-field theory for this model is shown in Fig. 1 for a cubic lattice that is experimentally most relevant. When there are equal proportions of each spin species, the Mott insulator becomes a pure antiferromagnetic or Néel state below a certain critical temperature. This state is characterized by a nonzero expectation value of the Néel order parameter vector n, also known as the staggered magnetization [11], which is related to the average on-site value of two neighboring spins by $\left\langle\hat{\mathbf{S}}_{A(B)}\right\rangle=\mathbf{m} \pm \mathbf{n}$. Here, we take the upper (lower) sign for the sublattice denoted by $A(B)$, and $\mathbf{m}=\mathbf{0}$ for the balanced case. Changing the proportion of each spin species gives rise to a nonzero average magnetization $\mathbf{m}=\left(0,0, m_{z}\right)$, where $m_{z}=S\left(N_{\uparrow}-N_{\downarrow}\right) /\left(N_{\uparrow}+N_{\downarrow}\right)$ is equal to the average spin per site of the system. Here, $S=1 / 2$ is the atomic pseudospin for the two-component Fermi mixture of interest and $N_{\uparrow, \downarrow}$ denotes the number of particles of each spin species corresponding to the two relevant hyperfine states. In contrast to the balanced case, the imbalanced system has a canted antiferromagnetic phase at low temperatures where the Néel vector is always perpendicular to the magnetization. Imbalance therefore breaks the full rotational symmetry of the system and 


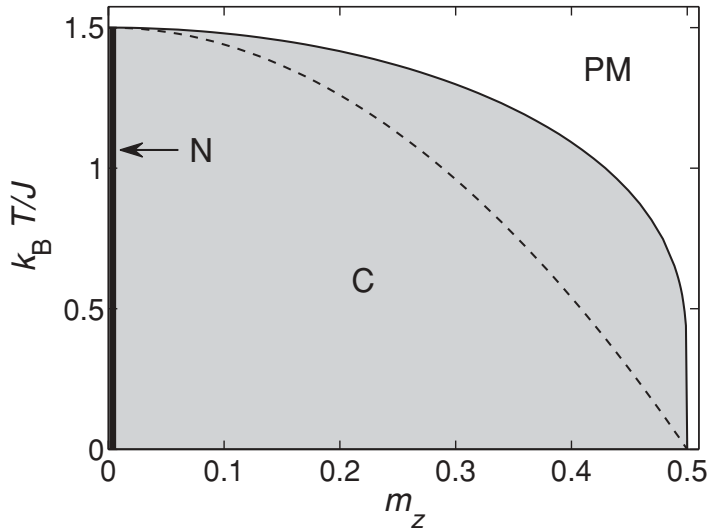

FIG. 1. Phase diagram of the imbalanced antiferromagnet in three dimensions. The solid curve is the critical temperature $k_{B} T / J$ for the canted antiferromagnet (C). Above the critical temperature the system is paramagnetic (PM). Here, $J$ is the superexchange coupling of the antiferromagnetic Heisenberg model. Also shown is the critical temperature for the corresponding Ising model (dashed line). In the absence of imbalance the system is in a pure antiferromagnetic or Néel (N) state.

defines an easy plane for the Néel vector that is perpendicular to the average magnetization.

Imbalance in the number of atoms of each spin is incorporated in the Heisenberg model by means of a constraint, which enforces that the average total spin is equal to the desired magnetization. This constraint considerably complicates the analysis compared to the balanced case where it is absent. Thus, the Hamiltonian for the imbalanced system is $\hat{H}=$ $(J / 2) \sum_{\langle i, j\rangle} \hat{\mathbf{S}}_{i} \cdot \hat{\mathbf{S}}_{j}-\sum_{i} \mathbf{B} \cdot\left(\hat{\mathbf{S}}_{i}-\mathbf{m}\right)$, where the effective magnetic field $\mathbf{B}$ acts as a Lagrange multiplier.

Within the usual Curie-Weiss mean-field analysis [11] we obtain the free energy per site

$$
\begin{aligned}
f(\mathbf{n}, \mathbf{m} ; \mathbf{B})= & \frac{J z}{2}\left(\mathbf{n}^{2}-\mathbf{m}^{2}\right)+\mathbf{m} \cdot \mathbf{B} \\
& -\frac{1}{2} k_{B} T \ln \left[4 \cosh \left(\frac{\left|\mathbf{B}_{A}\right|}{2 k_{B} T}\right) \cosh \left(\frac{\left|\mathbf{B}_{B}\right|}{2 k_{B} T}\right)\right],
\end{aligned}
$$

where $\mathbf{B}_{A(B)}=\mathbf{B}-J_{z} \mathbf{m} \pm J z \mathbf{n}, T$ is the temperature and $k_{B}$ is the Boltzmann constant. Here, $z=2 D$ is the number of nearest neighbours in the $D$-dimensional hypercubic lattice. The Lagrange multiplier $\mathbf{B}$ is then found from the constraint $\partial f(\mathbf{n}, \mathbf{m} ; \mathbf{B}) / \partial \mathbf{B}=\mathbf{0}$, Minimizing the free energy subject to this constraint, we find the critical temperature at which $\langle\mathbf{n}\rangle$ becomes nonzero to be $T_{c}=J z m_{z} /\left[2 k_{B} \operatorname{arctanh}\left(2 m_{z}\right)\right]$, corresponding to the solid curve in Fig. 1. At this point and throughout the antiferromagnetic phase, the constraint is solved by $\mathbf{B}=2 J z \mathbf{m}$ and $\langle\mathbf{n}\rangle$ is perpendicular to $\mathbf{m}$ in the minimum of the free energy. By contrast, in the Ising model where $\mathbf{n}$ and $\mathbf{m}$ are restricted to be parallel, one obtains $T_{c}=z J\left(1 / 4-m_{z}^{2}\right) / k_{B}$, which is the dashed line in Fig. 1.

In both the Néel and canted phases the low-energy excitations are spin waves called magnons. By linearizing the Heisenberg equations of motion of the on-site spin operators around their expectation values we obtain the magnon dispersion $\hbar \omega(\mathbf{k})=J z \sqrt{n^{2}\left(1-\gamma_{\mathbf{k}}^{2}\right)+m_{z}^{2}\left(1+\gamma_{\mathbf{k}}^{2}\right)}$, where $\gamma_{\mathbf{k}}=(2 / z) \sum_{i=1}^{D} \cos \left(d k_{i}\right)$ and $d$ is the lattice spacing. For the balanced situation the magnons have the usual antiferromagnetic dispersion that is doubly degenerate due to the presence of two transverse directions and linear for small momenta, as shown in Fig. 2. However, in the imbalanced case the nonzero average magnetization lifts this degeneracy. As a result, one of the dispersions becomes gapped corresponding to the Larmor precession of the spins around the effective magnetic field generated by the nonzero average magnetization. The gapped dispersion is quadratic at small momenta signaling the appearance of magnons with a ferromagnetic character.

In experiments the atomic gas is always confined in a trap. However, including a smooth harmonic trapping potential does not lead to inhomogeneities in the Mott-insulator state, but places a limit on the total number of particles beyond which the Mott insulator is destroyed [12]. As a result, the excitation spectrum shown in Fig. 2 becomes discretized in the trap due to finite-size effects. In the Mott-insulator phase, the system in a trap is thus essentially homogeneous and we may reasonably neglect the trap here [13].

\section{MERONS}

The long-wavelength dynamics obtained above can be summarized by the following nonlinear sigma model [14] with an action

$$
S[\mathbf{n}(\mathbf{x}, t)]=\int \mathrm{d} t \int \frac{\mathrm{d} \mathbf{x}}{d^{D}}\left\{\frac{1}{4 J z n^{2}}\left(\hbar \frac{\partial \mathbf{n}(\mathbf{x}, t)}{\partial t}-2 J z \mathbf{m} \times \mathbf{n}(\mathbf{x}, t)\right)^{2}-\frac{J d^{2}}{2}[\nabla \mathbf{n}(\mathbf{x}, t)]^{2}\right\} .
$$

Here, $\mathbf{n}(\mathbf{x}, t)$ is the local staggered magnetization at position $\mathbf{x}$ and time $t$ with fixed length $n$. The equilibrium value of the staggered magnetization is found from minimizing the Landau free energy, given by

$$
F[\mathbf{n}(\mathbf{x}), \mathbf{m}]=\int \frac{\mathrm{d} \mathbf{x}}{d^{D}}\left\{\frac{J d^{2}}{2}[\nabla \mathbf{n}(\mathbf{x})]^{2}+f[\mathbf{n}(\mathbf{x}), \mathbf{m}]\right\},
$$

where $f[\mathbf{n}(\mathbf{x}), \mathbf{m}]$ is the on-site free energy, which as we have seen, breaks the full rotational symmetry of the system in the presence of imbalance. Hence, the system is described by an $X Y$ model in the long-wavelength limit.

The equation of motion that follows from the nonlinear sigma model exhibits long-wavelength spin waves discussed previously, but also admits a class of topologically stable excitations due to the $X Y$ nature of the system. In the 


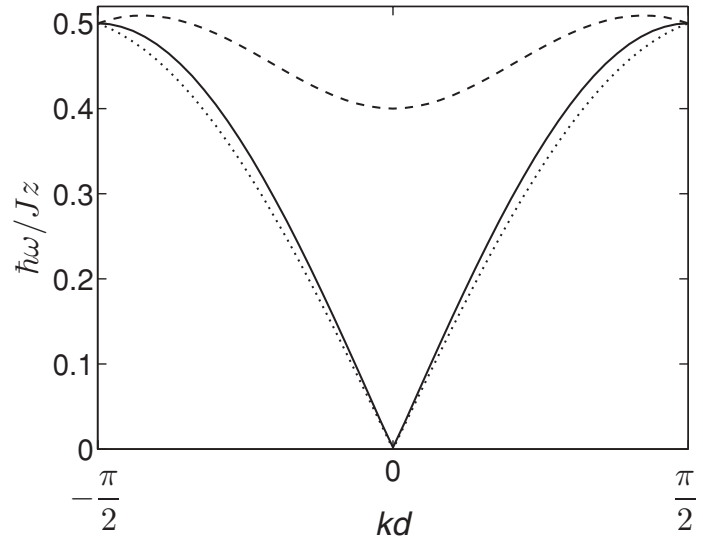

FIG. 2. The dispersion relation for the spin waves. Here, $\mathbf{k}=$ $k(1,1,1)$ is the wave vector. For zero magnetization (solid line), the dispersion is the usual doubly degenerate gapless antiferromagnetic dispersion. Imbalance splits the degeneracy and one of the dispersions (dashed line) becomes ferromagnetic and acquires a gap equal to $2 J_{z} m_{z}$, while the other remains antiferromagnetic (dotted line). The imbalanced dispersions shown here are for $m_{z}=0.2$.

three-dimensional case, these are coreless vortices with a spin component near the location of the vortex line that is out of the easy plane. In the two-dimensional case the topological excitations, known as merons $[15,16]$, are particularly interesting. Far away from the core of the meron, $\mathbf{n}(\mathbf{x})$ lies in the easy plane and forms a vortex with a vorticity or winding number of \pm 1 , while in the core region $\mathbf{n}(\mathbf{x})$ smoothly rotates either up or down out of the easy plane. This spin texture is characterized by two topological invariants, namely, the $X Y$ vorticity and the Pontryagin index, which counts how many times the texture $\mathbf{n}(\mathbf{x})$ winds around the unit sphere. The Pontryagin index is equal to $\pm 1 / 2$ corresponding to the upward or downward orientation of the core spin [17]. However, it does not play an important role for our purposes since, due to the symmetry of the action under the reflection $\mathbf{n} \rightarrow-\mathbf{n}$, merons differing only in their Pontryagin index have the same energy. Moreover, the interaction between two merons with a large separation is insensitive to the precise spin configuration in the core.

A meron spin texture in the $x-y$ plane with vorticity $n_{v}= \pm 1$ can be described by $\mathbf{n}=$ $\left\{\sqrt{n^{2}-\left[n_{z}(r)\right]^{2}} \cos \phi, n_{v} \sqrt{n^{2}-\left[n_{z}(r)\right]^{2}} \sin \phi, n_{z}(r)\right\}$, where $n=|\langle\mathbf{n}\rangle|$ and $\phi$ is the azimuth in the $x-y$ plane. Exact meron solutions that follow from minimizing the nonlinear sigma model action with this texture have an out-of-plane component that behaves as $n-n_{z}(r) \propto r^{2}$ near the origin and that decays exponentially far from the origin [18]. To determine the characteristic size of a meron, we perform a variational calculation substituting the spin texture of the meron into Eq. (2) for a range of temperatures and imbalance. A suitable variational ansatz that has the right properties near the origin but decays only as a power law for large $r$ is $n_{z}(r)=n /\left[(r / \lambda)^{2}+1\right]^{2}$. In this case, the energy of a single meron due to the gradient term in the nonlinear sigma model is $J n^{2} \pi\left[(511 / 60)-\pi-6 \ln 2+(1 / 2) \ln \left(A / \pi \lambda^{2}\right)\right]+\mathcal{O}\left(A^{-1}\right)$, which diverges as the logarithm of the area $A$ of the system. Incorporating the correct exponential decay for large distances changes the constant terms in this expression, but leaves the logarithm unaffected, showing that our ansatz is suitable to determine the characteristic core radius $\lambda$. Minimizing the sum of the gradient energy and the integrated on-site free energy in Eq. (1) of the meron texture leads to the characteristic core size $\lambda=d n \sqrt{\pi J / 2 F_{m}}$ shown in Fig. 3(b), with $F_{m}$ the integrated on-site free energy required for the formation of a meron spin texture of size $\lambda=d$. The Pontryagin index here is $+1 / 2$ and a texture with index $-1 / 2$ obtained by substituting $n_{z}(r) \rightarrow-n_{z}(r)$ will produce an identical variational result, as noted earlier. Also, the core structure will play no role in the interaction so long as the spacing of a pair of merons is larger than $\lambda$.

The energy of a single meron diverges logarithmically with the system area $A$ as $\left(J n^{2} \pi / 2\right) \ln \left(A / \pi \lambda^{2}\right)$. Thus, for the large system sizes of interest here, it is impossible to thermally excite a single meron below the critical temperature. This behavior is analogous to the divergence in the kinetic energy of a single vortex in a two-dimensional BEC. However, the energy of a pair consisting of a meron together with another meron of opposite vorticity, or antimeron, is finite since the topological deformation of the spin texture cancels far away from the center of the pair where the Néel vector always points in the same direction, as illustrated in Fig. 3(a).

Such meron-antimeron pairs are thermally excited below the critical temperature and are responsible for a KosterlitzThouless phase transition associated with the unbinding of the pairs. Indeed, the entropic contribution of the merons above the critical temperature is such that the system can lower its free energy through the proliferation of single merons. Topological

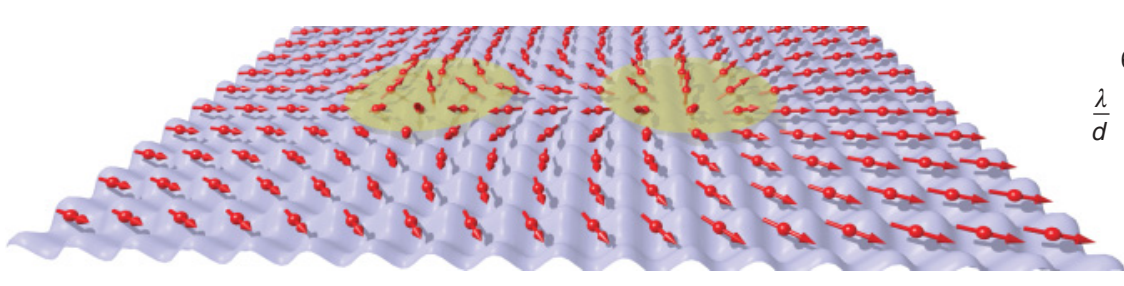

(a)

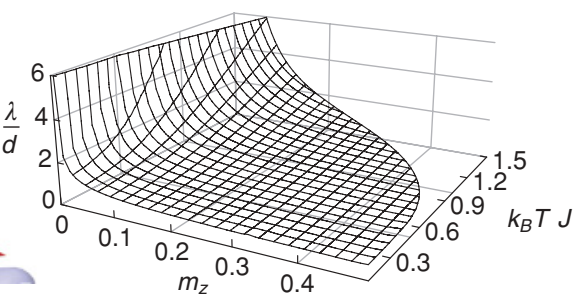

(b)

FIG. 3. (Color online) Merons in two dimensions. (a) A pair of merons with the same Pontryagin index but opposite vorticities. The arrows indicate the staggered magnetization, the periodic optical lattice potential is shown beneath, and the core regions of the merons are shown as yellow discs. (b) The radius of the core region $\lambda$ is plotted as a function of temperature and imbalance. The core size diverges as the imbalance is reduced to zero since full rotational symmetry is then restored and merons no longer exist. The surface terminates at the critical temperature where the system becomes paramagnetic. 


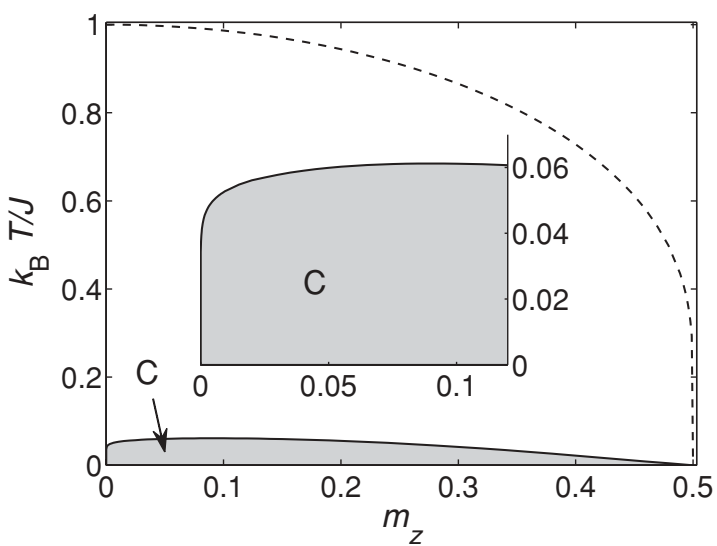

FIG. 4. Phase diagram of the imbalanced antiferromagnet in two dimensions. The critical temperature for the canted phase $(C)$ is drastically reduced compared to the mean-field result (dashed line) due to fluctuations and becomes a Kosterlitz-Thouless phase transition. Inset: a close-up of the phase transition for small imbalance.

excitations are notably absent from the mean-field analysis and as a result our mean-field analysis becomes even qualitatively incorrect in two dimensions. To correct for this, we used Monte Carlo results for an anisotropic O(3) model [19] to estimate the critical temperature for the Kosterlitz-Thouless transition from the canted antiferromagnet to the paramagnetic phase by making an analogy between the free energy contribution of our nonlinear sigma model and the anisotropy in the anisotropic $\mathrm{O}(3)$ model. We found that this transition occurs at a significantly lower temperature than predicted by mean-field theory in two dimensions, as shown in Fig. 4. In particular, the phase transition occurs at zero temperature for the balanced case where the system is rotationally symmetric. This is consistent with the Mermin-Wagner theorem for the Heisenberg model in two dimensions [20]. Although for $m_{z} \lesssim 0.2$ our anisotropy is more complicated than that used in the Monte Carlo simulations, we do not expect a rigourous calculation to deviate significantly from these results. In three dimensions, numerical studies yield only a $36 \%$ downward correction of the critical temperature in the balanced case [21] and topological excitations are not expected to significantly alter the phase diagram. For completeness, we mention here that in one dimension the imbalanced antiferromagnet is paramagnetic at any nonzero temperature [11].

\section{OUTLOOK AND CONCLUSION}

The imbalanced antiferromagnet can be readily investigated experimentally with modern techniques. Imbalance is achieved in an ultracold Fermi gas by driving hyperfine transitions with an rf field and a Néel-ordered state can be realized by, for example, adiabatically ramping up the optical lattice potential [12]. The Néel character and the Kosterlitz-Thouless transition can then be probed by the measurement of correlations in atom shot noise [22-24] or using Bragg reflection [25,26], which also enables spin waves to be examined. Merons also possess an internal Ising degree of freedom associated with their Pontryagin index that is very suggestive of their application as a qubit in topological quantum computation; this is a particularly interesting topic for further investigation.

\section{ACKNOWLEDGMENTS}

We are very grateful to Rembert Duine and Randy Hulet for providing useful comments and Niels Pannevis for his help with obtaining the results of the imbalanced Ising antiferromagnet. This work is supported by the Stichting voor Fundamenteel Onderzoek der Materie (FOM) and the Nederlandse Organisatie voor Wetenschaplijk Onderzoek (NWO).
[1] D. Jaksch, C. Bruder, J. I. Cirac, C. W. Gardiner, and P. Zoller, Phys. Rev. Lett. 81, 3108 (1998).

[2] M. P. A. Fisher, P. B. Weichman, G. Grinstein, and D. S. Fisher, Phys. Rev. B 40, 546 (1989).

[3] M. Greiner et al., Nature (London) 415, 39 (2002).

[4] M. Köhl, H. Moritz, T. Stoferle, K. Gunter, and T. Esslinger, Phys. Rev. Lett. 94, 080403 (2005).

[5] R. Jordens et al., Nature (London) 455, 204 (2008).

[6] U. Schneider et al., Science 322, 1520 (2008).

[7] A. Koetsier, D. B. M. Dickerscheid, and H. T. C. Stoof, Phys. Rev. A 74, 033621 (2006).

[8] M. W. Zwierlein, A. Schirotzek, C. H. Schunck, and W. Ketterle, Science 311, 492 (2006).

[9] G. B. Partridge et al., Science 311, 503 (2006).

[10] T. Gottwald and P. G. J. van Dongen, Phys. Rev. A 80, 033603 (2009).

[11] E. Fradkin, Field Theories of Condensed Matter Systems (Addison-Wesley, Redwood City, CA, 1991).

[12] A. Koetsier, R. A. Duine, I. Bloch, and H. T. C. Stoof, Phys. Rev. A 77, 023623 (2008).

[13] M. Snoek et al., New J. Phys. 10, 093008 (2008).

[14] S. Das Sarma, S. Sachdev, and L. Zheng, Phys. Rev. B 58, 4672 (1998).
[15] D. J. Gross, Nucl. Phys. B132, 439 (1978).

[16] I. Affleck, Phys. Rev. Lett. 56, 408 (1986).

[17] K. Moon, H. Mori, K. Yang, S. M. Girvin, A. H. MacDonald, L. Zheng, D. Yoshioka, and S. C. Zhang, Phys. Rev. B 51, 5138 (1995).

[18] S. Ghosh and R. Rajaraman, Int. J. Mod. Phys. B 12, 37 (1998).

[19] M. Klomfass, U. M. Heller, and H. Flyvbjerg, Nucl. Phys. B360, 264 (1991).

[20] N. D. Mermin and H. Wagner, Phys. Rev. Lett. 17, 1133 (1966).

[21] R. Staudt, M. Dzierzawa, and A. Muramatsu, Eur. Phys. J. B 17, 411 (2000).

[22] S. F. F. Gerbier et al., Nature (London) 434, 481 (2005).

[23] M. Greiner, C. A. Regal, J. T. Stewart, and D. S. Jin, Phys. Rev. Lett. 94, 110401 (2005).

[24] G. M. Bruun, O. F. Syljuasen, K. G. L. Pedersen, B. M. Andersen, E. Demler, and A. S. Sorensen, Phys. Rev. A 80, 033622 (2009).

[25] N. Fabbri, D. Clement, L. Fallani, C. Fort, M. Modugno, K. M. R. van der Stam, and M. Inguscio, Phys. Rev. A 79, 043623 (2009).

[26] D. Clément, N. Fabbri, L. Fallani, C. Fort, and M. Inguscio, Phys. Rev. Lett. 102, 155301 (2009). 